\title{
Left Ventricular Function Improve After Bench Press: A Speckle Tracking and 3D Echocardiography Study
}

Manuel Ruiz Bailen ${ }^{1 *}$, Jesus Cobo Molinos ${ }^{1}$, José Carlos Espada-Fuentes ${ }^{2}$, Ana María Castillo-Rivera ${ }^{3}$, Antonio Martínez-Amat ${ }^{2}$, María José Martínez-

Ramírez ${ }^{1}$, María Dolores Pola-Gallego-de-Guzmán ${ }^{4}$, Sigfredo Sánchez Ramos ${ }^{1}$ and José Ángel Ramos- Cuadra

${ }^{1}$ Departamento de Ciencias de la Salud. Universidad de Jaén. Complejo Hospitalario de Jaén

${ }^{2}$ Departamento de Ciencias de la Salud. Universidad de Jaén

${ }^{3}$ Intensive Care Medicine. Complejo Hospitalario de Jaén

${ }^{4}$ Intensive Care Medicine. Complejo Hospitalario de Torrecárdenas. Almería

*Corresponding author: Departamento de Ciencias de la Salud. Universidad de Jaén, Spain, Tel: (+34) 679 178994; E-mail: ruizbailen@gmail.com

Received date: October 13, 2016; Accepted date: October 23, 2016; Published date: October 31, 2016

Copyright: @ 2016 Ruiz-Bailen M, et al. This is an open-access article distributed under the terms of the Creative Commons Attribution License, which permits unrestricted use, distribution and reproduction in any medium, provided the original author and source are credited.

\begin{abstract}
Background: The speckle tracking echocardiography is a powerful tool that is expanding knowledge on cardiovascular physiology. At present fitness is an increasing sport, and the bench press is a typical exercise. The aim of this study was to investigate by speckle tracking echocardiography the systolic and diastolic properties of the left ventricle during bench press.

Methods: Interventional study. We perform an echocardiogram before, during and immediately after a bench press. During the bench press we assessed the changes suffered in systolic and diastolic functions. Speckle tracking parameter and 3D volume were calculated for left ventricle.

Results: In the study were included 59 male athletes with mean age $34.28 \pm 9.54$ years. Left ventricle ejection fraction is increased by 2D and 3D echocardiography [0.58 \pm 0.07 versus $0.62 \pm 0.07, p=0.017$.] The strain, strain rate, and systolic and diastolic velocities of longitudinal left ventricular fibers (by Speckle Tracking) are higher during exercise [(Strain $-21.1 \pm 4.09$ versus $-27.28 \pm 3.73, p<0.01$ ) (Strain Rate $-1.48 \pm 0,30$ versus $-2.30 \pm 0.57$; $p<0.0001]$. The $E / E^{\prime}$ ratio was unchanged but improved diastolic function improve by Speckle Tracking echocardiography. After the exercise there was more left intraventricular synchrony. Strength training degree could be associated with compliance and synchrony of left ventricle.
\end{abstract}

Conclusion: During bench press a discrete increase in ejection fraction and large variations in myocardial deformation occurs. Diastolic function is unchanged by 2D echocardiography. Strength training degree could be associated with compliance and synchrony of left ventricle.

Keywords: Speckle tracking; 3D echocardiography; Athlete; Bench press

\section{Abbreviations}

S: Strain; SR: Strain Rate; LVEF: Left Ventricular Ejection Fraction; EDLVV: End Diastolic Left Ventricular Volume; ESLVV: End Systolic Left Ventricular Volume; VTI: Velocity Time Integral; SRS: Systolic Strain Rate; 2D: Two Dimensional; 3D: Four Dimensional; DTI: Doppler Tissue Imaging; RM: Repetition Maximal; IPAQ: International Physical Activity Questionnaires; ESC: European Society of Cardiology; PCWP: Pulmonary Capilar Wedge Pressure; VP: Mitral Flow Velocities Propagation; ASE: American Society of Echocardiography

\section{Introduction}

Speckle tracking echocardiography is introducing new hypotheses about the pathophysiology of cardiovascular elite athlete. The Speckle Tracking allows us to evaluate the strain (S), the strain rate (SR), tissue velocities and displacement; being it less influenced by the angle of study, or by the load nor sex, nor they age or systolic blood pressure values [1,2]. Ventricular remodeling that athlete produce is very variable, depending on the type of sport, age, gender, time or type of training, race or genetics [3]. We consider that there are two types of remodeled, concentric and eccentric.

Probably the most important remodeling was a mixture of both being generated by the adaptation of the myocardial cells. There may be a relationship between training degrees with myocardial performance. In a healthy person is assumed that in an exercise which is trained systolic and diastolic function be improved, increasing ejection fraction and left ventricular compliance. This could lead to an increase in Strain, Strain rate, peak systolic and diastolic velocities and displacement of left ventricular fibers [3].

Usually, studies are performed on elite athletes having completed the exercise. The fitness is a growing sport, and bench press exercise is very representative. However there are few studies on no elite, and is not known the immediate performance of muscle fibers during exercise, especially while strength training by bench press. With this study we evaluate the peak speeds, S, SR and displacement of left ventricular longitudinal muscle fibers in the bench press exercise. We also want to assess whether there is any relationship between degrees 
Page 2 of 6

of strength training with echocardiographic parameters of myocardial performance.

\section{Methods}

\section{Study Design}

It is a cross-sectional study, where measurements were carried out before, during and immediately after exercise. Study was carried in gym "Emotion Sport Club of Jaén - http://emotionsportsclub.com/-" It belongs to PAIDI CTS 606 Andalusian Health Service Project No. PI-0585-2012, approved by the local ethics committee, and funded by the department of Health of the Government of Andalusia, Government Andalusia, Spain.

Inclusion phase was conducted during the last week of July 2013. We included male athletes between 25 and 40 years. We define athletes that: have a high level of physical activity (quantitative value $>3000$ meets / min per week) in international Physical Activity Questionnaires (IPAQ) (Spanish version 2002).

\section{Exclusion criteria}

- Athletes were excluded with any known cardiovascular disease or detected during the study

- Athletes with poor sound quality

- Athletes who do not wish to be included in the study, and therefore did not sign the Informed Consent

- Previous arrhythmias diagnosis

- Previous hypertension diagnosis

\section{Intervention and clinical cohort}

All patients meeting inclusion criteria were performed transthoracic echocardiography being recorded in high quality digital format (using acoustic catches) for further analysis "off line by Syngo software program. U.S. Siemens $2013^{\circ}$. Echocardiographic data were evaluated blindly (time and type of athlete will be unknown). Similarly were conducted three separate studies and will be assessed the degree of inter observer agreement.

\section{Image acquisition and processing}

Standard transthoracic echocardiograms were performed using a commercially available system (SC2000, Siemens ${ }^{\bigotimes}$. U.S.). Transthoracic examinations were carried in the decubitus supine position. For the acquisition of left ventricular functional data was used the apical fourchamber orientation. Region of interest were manually traced along the endomyocardial border in the six segments. We adopted a six segments model to assess regional and global left ventricular performance in the longitudinal direction. The frame rate were as high as possible (70-120 f/s), with multiple focal point. All images were optimized with gain, compression, and dynamic range images. We use the probes $4 \mathrm{~V} 1 \mathrm{C}$, and $4 \mathrm{Zlc}$. Off-line analysis was performed blinded by Syngo software, U.S. Siemens ${ }^{\bigotimes}$. 2013. We evaluated the usual echocardiographic parameters of American Society of Echocardiography. We calculate left ventricular ejection fraction (LVEF) by $2 \mathrm{D}$ and $3 \mathrm{D}$ volume, quantification of the E/E', estimating the PCWP and parameters derived from speckle tracking, such as S, SR, displacement, longitudinal and radial velocities in left ventricle.

\section{Exercise stress-echo}

It is a cross-sectional study, in which the measurements were carried out before, during and after exercise. Degree of strength training will be assessed by the maximum load for 1RM. The following protocol was used to evaluate the strength and prevent injuries:

- Start with a warm-up set with a light load allowing you to perform 10 repetitions

- Rest for one minute

- Perform another series with a resistance heating that will allow complete 3-5 repetitions. This usually means increased $5-10 \%$ of the previous set

- Rest for two minutes

- Estimate another increase (5-10\%) that allowed you to fully 2 or 3 repetitions

- Rest for four minutes

- Estimate another increase (5-10\%) that allows for a single repetition of the exercise

- Break four minutes and then calculate a new moderate increase in weight (5-10\%), and repeat the test

- If you cannot lift the weight, after the break four minutes, the weight ( 2.5 to $5 \%)$ will be reduced, and then repeat

Continue increasing or decreasing the weight as necessary to determine their repetition maximum (RM) real. After determining the training load using the $1 \mathrm{RM}$ test, the load can move a total of 10 replicates were estimated. Training protocol is based on the 10 sets of 10 repetitions with a load current of $75 \%$ of $1 \mathrm{RM}$ estimating, each with a break of 1'30 "between sets, during the conduct of the exercise, series and rest included, perform echocardiography [4].

\section{Statistical Analysis}

A study for quantitative variables was performed using ANOVA. For variables that did not follow a normal probability distribution was used the means test for nonparametric samples. Their results were presented using means and standard deviations. It was regarded a $\mathrm{p}$ value $<0.05$ statistically significant. The correlation between LVEF $2 \mathrm{D}$ and $3 \mathrm{D}$ were evaluated using the Spearman test and linear regression. Were also conducted bivariate correlations enters the weight of the 1 RM with echocardiographic parameters using the Spearman test. We were used correlations between variables by Pearson's $r$ coefficient for variability intra observer.

\section{Results}

63 male Caucasian athletes were included in the study; one was excluded for developing atrial tachycardia diagnosed disease WolffParkinson-White. Mean age were $34.28 \pm 9.54$ years. RM values were: mean $97.51 \mathrm{Kg}$; median $100.6 \pm 31.39 \mathrm{Kg}$. Intra observer basal variability was 0.861 for 2D-LVEF; 0.878 in S 0.861 for SR and 0.784 for systolic peak longitudinal velocity (all $\mathrm{p}<0.0001$ ).

\section{Left ventricular systolic function}

After completing the exercise a discrete increase in LVEF was observed by volumetric 2 and 3D echocardiographs (Tables 1 and 2). Likewise, a higher volumetric increase is detected in the apical segments, apicolateral specially. The correlation coefficient between LVEF by 2 and 3D was 0.446 (Spearman), with $p=0.028$. By linear regression and an R2 of 0.733 and $\mathrm{p}=0.016$ is observed. End-diastolic 
Citation: Ruiz-Bailen M, Cobo-Molinos J, Espada-Fuentes JC, Castillo-Rivera AM, Martínez-Amat A, Martínez-Ramírez MJ, Pola-Gallego-deGuzmán MD, Sánchez-Ramos S and Ramos-Cuadra JA (2017) Left Ventricular Function Improve After Bench Press: A Speckle Tracking and 3D Echocardiography Study. J Sports Med Doping Stud 7: 186. doi:10.4172/2161-0673.1000186

Page 3 of 6

volumes increase very slightly, without modifying the sphericity index (0.6). The tissue Doppler image shows increase septal and lateral systolic velocities "s" of the mitral annulus.

\begin{tabular}{|c|c|c|c|}
\hline \multicolumn{4}{|l|}{ Transmitral Flow } \\
\hline & Basal & After bench press & P-value \\
\hline Sphericity index & $(n=35) 0.74 \pm 0.14$ & $(n=30) 0.84 \pm 0.097$ & 0.580 \\
\hline Peak velocity $E$ wave $(\mathrm{m} / \mathrm{s})$ & $(n=38) 0.86 \pm 0.25$ & $(n=36) 1.10 \pm 0.21$ & 0.000 \\
\hline Peak velocity $A$ wave $(\mathrm{m} / \mathrm{s})$ & $(n=37) 0.47 \pm 0.15$ & $(n=36) 0.78 \pm 0.18$ & 0.000 \\
\hline E deceleration time (ms) & $(n=37) 204 \pm 54$ & $(n=35) 205 \pm 56$ & 0.849 \\
\hline IVRT (ms) & $(n=37) 86.3 \pm 19.5$ & $(n=35) 73.5 \pm 16.7$ & 0.001 \\
\hline Transmitral VTI (m) & $(n=37) 0.22 \pm 0.043$ & $(n=36) 0.23 \pm 0.051$ & 0.301 \\
\hline Tei Index & $(n=31) 0.38 \pm 0.13$ & $(n=32) 0.33 \pm 0.11$ & 0.62 \\
\hline
\end{tabular}

Table 1: Diastolic basal to after bench press parameters.

\begin{tabular}{|l|l|l|l|}
\hline \multicolumn{2}{|l|}{ Mitral Tisular Doppler Image } & Basal & After bench press \\
\hline$(\mathrm{m} / \mathrm{s})$ & $(n=38) 0.14 \pm 0.037$ & $(n=38) 0.19 \pm 0.04$ & P-value \\
\hline E'septal & $(n=34) 0.21 \pm 0.031$ & $(n=38) 0.23 \pm 0.04$ & 0.000 \\
\hline E'Lateral & $(n=37) 0.11 \pm 0.03$ & $(n=37) 0.16 \pm 0.053$ & 0.000 \\
\hline S septal & $(n=34) 0.13 \pm 0.03$ & $(n=37) 0.23 \pm 0.056$ & 0.000 \\
\hline S Lateral & $(n=36) 0.09 \pm 0.02$ & $(n=37) 0.13 \pm 0.05$ & $(n=36) 0.12 \pm 0.047$ \\
\hline A' septal & $(n=34) 0.09 \pm 0.09$ & 0.000 & 0.559 \\
\hline A' Lateral & &
\end{tabular}

Table 2: Mitral tisular doppler image.

\section{Diastolic parameters}

Table 3 shows diastolic parameters. Pulsed Doppler transmitral flow shows a discrete increment of the $\mathrm{E}$ wave velocity, whereas diminished duration of peak med-diastolic wave velocity. Also a clear increase in wave velocities E'septal and lateral occurs. However not change the E / E' ratio. Similarly no were significant changes in the VP or filling of the pulmonary veins. All this is consistent with the absence of modification of the PCWP.

\begin{tabular}{|c|c|c|c|}
\hline \multicolumn{4}{|c|}{ Pulmonary Veins Filling flow } \\
\hline & Basal & After bench press & P-value \\
\hline Velocity S (m/s) & $(n=45) 0.51 \pm 0.16$ & $(n=31) 0.56 \pm 0.13$ & 0.146 \\
\hline Velocity $D(\mathrm{~m} / \mathrm{s})$ & $(n=45) 0.56 \pm 0.13$ & $(n=31) 0.57 \pm 0.19$ & 0.780 \\
\hline S VTI & $(n=31) 0.14 \pm 0.072$ & $(n=21) 0.14 \pm 0.12$ & 0.912 \\
\hline D VTI & $(n=30) 0.16 \pm 0.089$ & $(n=21) 0.24 \pm 0.23$ & 0.104 \\
\hline Systolic fraction filling & $(n=37) 0.49 \pm 0.47$ & $(n=31) 0.13 \pm 0.7$ & 0.748 \\
\hline Velocity AR & $(n=28) 0.27 \pm 0.078$ & $(n=18) 0.46 \pm 0.15$ & 0.00 \\
\hline Deceleration $\mathrm{D}$ wave time & $(n=30) 267.9 \pm 113.7$ & $(n=18) 236 \pm 76.4$ & 0.298 \\
\hline
\end{tabular}

Table 3: Pulmonary veins filling flow. 
Citation: Ruiz-Bailen M, Cobo-Molinos J, Espada-Fuentes JC, Castillo-Rivera AM, Martínez-Amat A, Martínez-Ramírez MJ, Pola-Gallego-deGuzmán MD, Sánchez-Ramos S and Ramos-Cuadra JA (2017) Left Ventricular Function Improve After Bench Press: A Speckle Tracking and 3D Echocardiography Study. J Sports Med Doping Stud 7: 186. doi:10.4172/2161-0673.1000186

Page 4 of 6

\section{Speckle tracking parameters}

Global Peaks systolic longitudinal velocities were increased and also they increased in all segments except the segment apicoseptal (the increase was not statistically significant) (Figures 1-4). Interestingly, the normalized heart rate delay of systolic peak velocities between basal and lateral segments was decreased which may suggest an improvement in ventricular synchrony. Early longitudinal diastolic velocity (E) was decreased during exercise), suggestive of increased left ventricular compliance. Left ventricular radial velocities were modified similarly to the longitudinal. S, SR and segmental left ventricular also suffered average systolic and diastolic increase (Tables 4 and 5) (Figures 5 and 6). Similarly, despite absence of changes in the E/E ', we observed changes in relationships S/SR, IVRT/SR and IVRT/SR ratio suggestive of improvement in left ventricular compliance during exercise. We have obtained a significant correlation between the maximum load weight of $1 \mathrm{RM}$ with
- Septal wave E' velocity (correlation coefficient $0,247, \mathrm{p}=0.010$ )

- Pulmonary vein systolic VTI (correlation coefficient $0.215, \mathrm{p}=046$ )

- The delay of peak systolic velocities of longitudinal fibers (correlation coefficient $-0.273 ; \mathrm{p}=0.004$ )

- Mid-diastolic velocities of longitudinal fibers (correlation coefficient 0.193, p=.005)

- Radial velocities of the $\mathrm{E}$ and $\mathrm{A}$ waves (correlation coefficient $0.295, \mathrm{p}=0.003$ and $0.287, \mathrm{p}=0.006$ respectively)

- Mid-diastolic strain (correlation coefficient -0.255; $\mathrm{p}=0.010$ )

- Peak systolic SR delay (correlation coefficient - 476; $\mathrm{p}=0.0001$ )

- Longitudinal displacement (correlation coefficient $0.278 ; \mathrm{p}=0.038$ )

- Longitudinal displacement delay time (correlation coefficient $0.433 ; \mathrm{p}=0.002)$

- Longitudinal mid-diastolic displacement (correlation coefficient $0.398 ; \mathrm{p}=0.004)$.

Left Ventricular Ejection Fraction

\begin{tabular}{|l|l|l|l|}
\hline & Basal & Press bench & P-value \\
\hline LVEDV $(m L) 3 D$ & $(n=33) 117 \pm 28.4$ & $(n=33) 113 \pm 26$ & 0.284 \\
\hline LVESV 3D $(m L)$ & $(n=33) 54.2 \pm 18.66$ & $(n=33) 41 \pm 14.7$ & 0.017 \\
\hline MAPSE & $(n=56) 19.17 \pm 3.93$ & $(n=47) 21.29 \pm 3.67$ & 0.006 \\
\hline LVEF 3D & $(n=51) 0.58 \pm 0.07$ & $(n=52) 0.62 \pm 0.07$ & 0.017 \\
\hline LVEF 2D & $(n=56) 0.57 \pm 0.77$ & $(n=49) 0.63 \pm 0.78$ & 0.000 \\
\hline
\end{tabular}

Table 4: Left ventricular ejection fraction.

\begin{tabular}{|c|c|c|c|}
\hline \multicolumn{4}{|l|}{ Speckle Tracking } \\
\hline & Basal & Press bench & P-value \\
\hline Peak systolic longitudinal velocity $(\mathrm{cm} / \mathrm{s})$ & $(n=36) 4.29 \pm 1.10$ & $(n=31) 6.10 \pm 2.19$ & 0.000 \\
\hline LV longitudinal velocity delay (anterior-lateral walls) ms & $(n=36) 122.33 \pm 52.92$ & $(n=28) 78.5 \pm 64.79$ & 0.055 \\
\hline LV longitudinal radial velocity & $(n=36) 2.70 \pm 0.98$ & $(n=31) 3.79 \pm 1.54$ & 0.000 \\
\hline LV longitudinal radial delay (ms) & $(n=35) 125 \pm 48.35$ & $(n=27) 118 \pm 44.58$ & 0.412 \\
\hline LV longitudinal Strain & $(n=36)-21.1 \pm 4.09$ & $(n=31)-27.28 \pm 3.73$ & 0.000 \\
\hline LV longitudinal Strain delay (ms) & $(n=35) 109 \pm 76.03$ & $(n=28) 93.5 \pm 63.16$ & 0.506 \\
\hline LV longitudinal Strain E & $(n=3)-19.28 \pm 2.34$ & $(n=1)-25 \pm 1.37$ & 0.001 \\
\hline LV Strain Rate & $(n=36)-1.48 \pm 0.30$ & $(n=31)-2.30 \pm 0.57$ & 0.000 \\
\hline LV Strain Rate E & $(n=33) 1.21 \pm 0.48$ & $(n=27) 1.83 \pm 0.77$ & 0.016 \\
\hline LV Strain Rate A & $(n=33) 0.47 \pm 0.34$ & $(n=27) 0.67 \pm 0.95$ & 0.191 \\
\hline S/Strain rate & $(n=39) 13.2 \pm 3.29$ & $(n=35) 11.6 \pm 1.95$ & 0.000 \\
\hline IVRT/ SR & $(n=54)-64.43 \pm 17.48$ & $(n=47)-33.51 \pm 10.46$ & 0.000 \\
\hline
\end{tabular}

Table 5: Speckle tracking.

\section{Discussion}

Sport provides clear benefits. Elite athletes could have higher tissue velocities and strain. But most athletes are not elite athletes. On the other hand most studied sports are soccer, cycling, running, using young athletes. Despite increase in fitness there are no studies on the changes that occur during training on the longitudinal fibers while 
Citation: Ruiz-Bailen M, Cobo-Molinos J, Espada-Fuentes JC, Castillo-Rivera AM, Martínez-Amat A, Martínez-Ramírez MJ, Pola-Gallego-deGuzmán MD, Sánchez-Ramos S and Ramos-Cuadra JA (2017) Left Ventricular Function Improve After Bench Press: A Speckle Tracking and 3D Echocardiography Study. J Sports Med Doping Stud 7: 186. doi:10.4172/2161-0673.1000186

Page 5 of 6

bench pressing is performed in amateur athletes. In the present study we found that there is a slight increase in LVEF obtained by 2 and 3D. However we have obtained a clear increase of velocities and deformity. This reflects a clear contractile improvement is not fully explained by the load, in fact we have not achieved significant changes in comprehensive speeds time of ventricular filling, or ventricular geometry. The increase of S and SR has been described in other sports [5,6] even without detecting changes in LVEF [7]. Although it is accepted that an increase of S and SR occurs during exercise in elite athletes today do not know the behavior of the myocardial fibers in the non-elite athletes [8-11].

Although we have not obtained changes in diastolic function, we found by Speckle Tracking signs suggestive of a possible improvement in diastolic function and a potential increase in left ventricular compliance. Acar et al. [12] obtained improved S in the left atrium. These findings may explain the lack of variability in the E/E 'and PCWP [13].

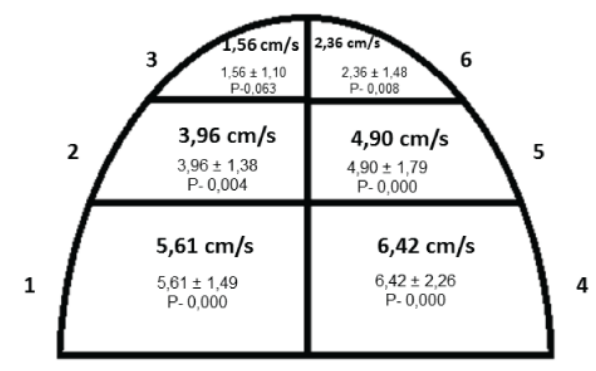

Segmentary Longitudinal peak systolic Velocity basal

Figure 1: Segmentary longitudinal peak systolic velocity basal.

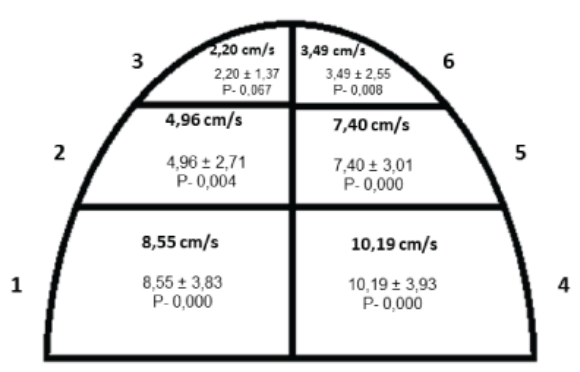

Segmentary longitudinal peak systolic velocity during press bench

Figure 2: Segmentary longitudinal peak systolic velocity during press bench.

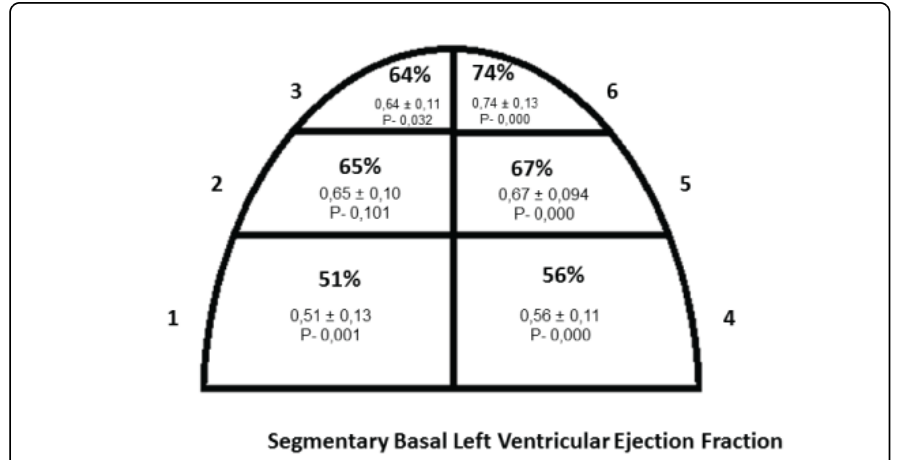

Figure 3: Segmentary basal left ventricular ejection fraction.

This could suggest that fitness may improve diastolic function in patients with heart disease. [13-15]. In preclinical studies is demonstrate that cardiovascular adaptations to exercise are dependent intensity and that could change the molecular architecture of the cardiomyocyte. Kemi et al. [16] demonstrated that cardiovascular adaptations to training are intensity-dependent. A close correlation between $\mathrm{VO}_{2} \mathrm{max}$, cardiomyocyte dimensions and contractile capacity suggests significantly higher benefit with high intensity, whereas endothelial function appears equivalent at moderate levels. Thus, exercise intensity emerges as important variable in future preclinical and clinical investigations. This group also detects that exercise training remodel the hearts [17].

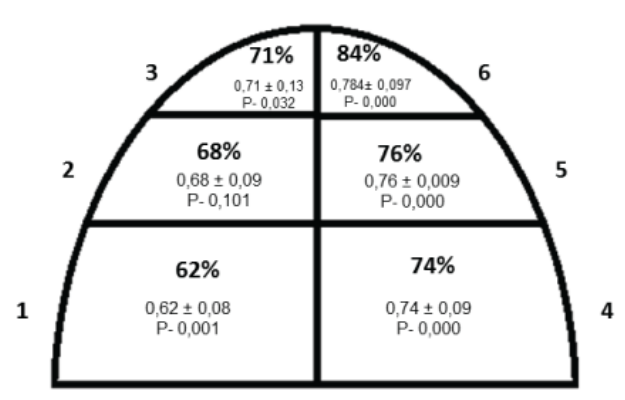

Segmentary Left Ventricular Ejection Fraction during Press Bench

Figure 4: Segmentary left ventricular ejection fraction during press bench.

This would also have important clinical implications, could be assessed by cardiac imaging or changes in intra ventricular pressure. So actually there is no evidence that strength training using bench press modify contractility and left ventricular compliance. This study indicates that training degree beneficially can improve left ventricular compliance. This could explain the clinical improvement detected by high levels of muscular strength appear to protect hypertensive men and woman against all-cause mortality [18-21]. This study raises a new question we could exercise improve cardiac synchrony? This could also have an important clinical applicability implying an increased cardiac output. 
Citation: Ruiz-Bailen M, Cobo-Molinos J, Espada-Fuentes JC, Castillo-Rivera AM, Martínez-Amat A, Martínez-Ramírez MJ, Pola-Gallego-deGuzmán MD, Sánchez-Ramos S and Ramos-Cuadra JA (2017) Left Ventricular Function Improve After Bench Press: A Speckle Tracking and 3D Echocardiography Study. J Sports Med Doping Stud 7: 186. doi:10.4172/2161-0673.1000186

Page 6 of 6

\section{Limitations}

This is a pilot study with a low samples and only show possibility of practicing hypothesis. It was done in gym, and results should be confirmed. There may be variability in relation to age, and a possible bias related to sex, but the speckle tracking is independent sample of those situations. In any case we believe that this age could be closer to reality than those in young elite athletes.

\section{Conclusion}

During bench press to systolic and diastolic Increase discrete functions. Speckle tracking is more sensitive than the $2 \mathrm{D}$ detect changes in myocardial performance suffered. Intensity strength training might correlate with improved parameters of diastolic function.

\section{Acknowledgements}

I want to thank my Kendo and Iaido "Juan Antonio Coronil Moreno" Sensei all his contribution so that I regained my confidence in myself. I also wish to sincerely thank Mr. Urbano Ríos and Pablo Puertas, for making possible the realization of this study. Similarly Siemens U.S and Spain want to thank them for leaving free echocardiography equipment high-end gym. And finally the Emotiont's Club Jaén and its users for allowing use of their facilities and their users paid to it.

\section{Funding}

Servicio Andaluz de Salud. SAS Project no PI-0585-2012. Approved by the local ethics committee, and funded by the "Consejería de Salud" of the Government of Andalusia. Spain. PAIDI CTS-606.

\section{References}

1. Perk G, Tunick PA, Kronzon I (2007) Non-Doppler two-dimensional strain imaging by echocardiography-from technical considerations to clinical applications. J Am Soc Echocardiogr 20: 234-243.

2. Marwick TH, Leano RL, Brown J, Sun JP, Hoffmann R, et al. (2009) Myocardial strain measurement with 2-dimensional speckle-tracking echocardiography: definition of normal range. JACC Cardiovasc Imaging 2: 80-84.

3. Baggish AL, Yared K, Weiner RB, Wang F, Demes R, et al. (2010) Differences in cardiac parameters among elite rowers and subelite rowers. Med Sci Sports Exerc 42: 1215-1220.

4. Brzycki M (1993) Strength Testing-Predicting a One-Rep Max from Reps-to-Fatigue. J Health Phys Ed Rec and Dance 64: 88-90.

5. Monte IP, Mangiafico S, Buccheri S, Bottari VE, Lavanco V, et al. (2015) Myocardial deformational adaptations to different forms of training: a real-time three-dimensional speckle tracking echocardiographic study. Heart Vessels 30: 386-395.

6. Schattke S, Xing Y, Lock J, Brechtel L, Schroeckh S, et al. (2014) Increased longitudinal contractility and diastolic function at rest in welltrained amateur Marathon runners: a speckle tracking echocardiography study 12: $11 \mathrm{pp}$.
7. Dalen H, Thorstensen A, Aase SA, Ingul CB, Torp H, et al. (2010) Segmental and global longitudinal strain and strain rate based on echocardiography of 1266 healthy individuals: the HUNT study in Norway. Eur J Echocardiogr 11: 176-183.

8. De Luca A, Stefani L, Pedrizzetti G, Pedri S, Galanti G (2011) The effect of exercise training on left ventricular function in young elite athletes. Cardiovasc Ultrasound 9: 27pp.

9. Simsek Z, Tas MH, Degirmenci H, Yazıcı AG, Ipek E, et al. (2013) Speckle tracking echocardiographic analysis of left ventricular systolic and diastolic functions of young elite athletes with eccentric and concentric type of cardiac remodeling. Echocardiography 30: 1202-1208.

10. Santoro A, Alvino F, Antonelli G, Cameli M, Bertini M, et al. (2015) Left Ventricular Strain Modifications after Maximal Exercise in Athletes: A Speckle Tracking Study. Echocardiography 32: 920-927.

11. Vitarelli A, Capotosto L, Placanica G, Caranci F, Pergolini M, et al. (2013) Comprehensive assessment of biventricular function and aortic stiffness in athletes with different forms of training by three-dimensional echocardiography and strain imaging. Eur Heart J Cardiovasc Imaging 14:1010-1020.

12. Acar RD, Bulut M, Ergun S, Yesin M, Akçakoyun M (2015) Evaluation of the Effect of Cardiac Rehabilitation on Left Atrial and Left ventricular Function and Its Relationship with Changes in Arterial Stiffness in Patients with Acute Myocardial Infarction. Echocardiography 32: 443447.

13. Esfandiari S, Sasson Z, Goodman JM (2014) Short-term high-intensity interval and continuous moderate-intensity training improve maximal aerobic power and diastolic filling during exercise. Eur J Appl Physiol 114: 331-343.

14. Haykowsky M, Humen D, Teo K, Quinney A, Souster M, et al. (2000) Effects of 16 weeks of resistance training on left ventricular morphology and systolic function in healthy men $>60$ years of age. Am J Cardiol 85: 1002-1006.

15. Adams J, Schmid J, Parker RD, Coast JR, Cheng D, et al. (2014) Comparison of force exerted on the sternum during a sneeze versus during low-, moderate-, and high-intensity bench press resistance exercise with and without the valsalva maneuver in healthy volunteers. Am J Cardiol 113: 1045-1048.

16. Kemi OJ, Haram PM, Loennechen JP, Osnes JB, Skomedal T, et al. (2005) Moderate vs. high exercise intensity: differential effects on aerobic fitness, cardiomyocyte contractility, and endothelial function. Cardiovasc Res 67: 161-172.

17. Kemi OJ, Hoydal MA, Macquaide N, Haram PM, Koch LG, et al. (2011) The effect of exercise training on transverse tubules in normal, remodeled, and reverse remodeled hearts. J Cell Physiol 226: 2235-2243.

18. Dos Santos ES, Asano RY, Gomes Filho I, Nilson Lima L, Panelli P, et al. (2014) Acute and Chronic Cardiovascular Response to 16 Weeks of Combined Eccentric or Traditional Resistance and Aerobic Training in Elderly Hypertensive Women: A Randomized Controlled Trial. J Strength Cond Res 28: 3073-3084.

19. Gerage AM, Forjaz CL, Nascimento MA, Januário RS, Polito MD, et al (2013) Cardiovascular adaptations to resistance training in elderly postmenopausal women. Int J Sports Med 34: 806-813.

20. Cunha RM, Jardim PC (2012) Subacute blood pressure behavior in elderly hypertensive women after resistance exercise session. J Sports Med Phys Fitness 52: 175-180.

21. Artero EG, Lee DC, Ruiz JR, Sui X, Ortega FB, et al. (2011) A prospective study of muscular strength and all-cause mortality in men with hypertension. J Am Coll Cardiol 57: 1831-1837. 